\title{
Diagnosis of Hepatocellular Carcinoma with Non-Invasive Imaging: a Plea for Worldwide Adoption of Standard and Precise Terminology for Describing Enhancement Criteria
}

\author{
Diagnose des hepatozellulären Karzinoms mittels nicht-invasiver Bildgebung: Ein Plädoyer \\ für die weltweite Umsetzung einer standardisierten und präzisen Fachterminologie zur \\ Beschreibung des Enhancements
}

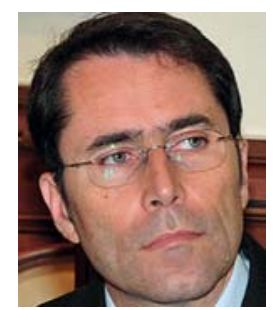

Fabio Piscaglia

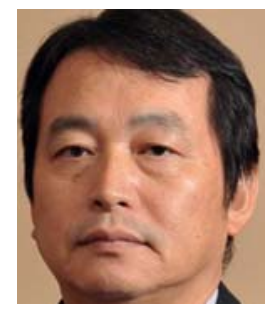

Masatoshi Kudo

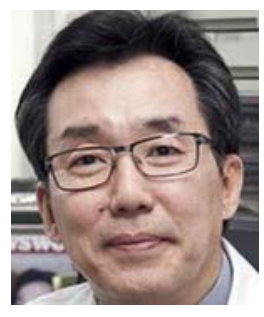

Kwang-Hyub Han

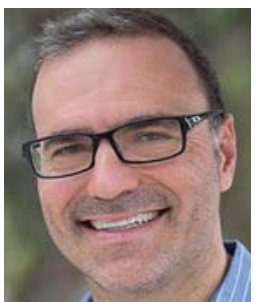

Claude Sirlin
Correspondence

Prof. Fabio Piscaglia

Dpt of Medical and Surgical Sciences DIMEC Unit of Internal Medicine via Albertoni 15

40138 Bologna

Italy

fabio.piscaglia@unibo.it
Bibliography

DOI http://dx.doi.org/10.1055/s-0042-124204

Published online: 2017 | Ultraschall in Med 2017; 38: 9-11

(c) Georg Thieme Verlag KG Stuttgart · New York

ISSN 0172-4614
Hepatocellular carcinoma in cirrhosis is unique in oncology since a definitive diagnosis based on non-invasive imaging can be used without biopsy confirmation to determine even the most radical therapies such as liver transplantation. Given the enormous responsbility assigned to non-invasive imaging in guiding HCC management one would expect that there would be standardized terminology in use throughout the world for describing the diagnostic imaging criteria. Surprisingly, this is not the case. Different guidelines have adopted different terminology for assessing the temporal enhancement characteristics of HCC. The adoption of different terminology by different guidelines, even if intended to have similar meaning, contributes to inconsistency in clinical care, causes confusion in research, and is a barrier to progress in the field.

It is time to standardize the terminology for describing temporal enhancement criteria, which will be suggested at the end of the present editorial in line with recently published relevant documents in this field.

A summary of the current guidelines and of the variance of the terminology is useful to understand the potential causes of variability and misunderstandings. Guidelines released by the AASLD in 2005 [1] recognized the need to incorporate imaging features from both the arterial phase and the subsequent venous/delayed phase. This was done to help avoid false-positive diagnosis of perfusion disturbances caused by arterioportal shunting which also can manifest with hyperenhancement in the arterial phase. The criteria for the diagnosis of HCC was a lesion showing "arterial hypervascularity that washes out in the early or delayed venous phase" [1]. The update of these guidelines in 2011 adopted the wording of "intense arterial uptake followed by wash out in the venous-delayed phases" [2]. Although the AASLD guidelines should be lauded for recognizing the importance of "washout", the terminology advocated by the AASLD is suboptimal. The term "hypervascularity" is ambiguous as it encompasses multiple different pathophysiological alterations including number and size of vessels, type of vessels, quality of their walls (fenestrated or not fenestrated sinusoids, capillarization), degree of vascular tone, etc. - most of which cannot be assessed reliably by imaging. A descriptive term would be more appropriate. In this sense, the usage of "intense arterial uptake" in the 2011 update is an improvement [2], but remains suboptimal for several reasons. In a liver with only arterial inflow and no portal perfusion (e. g. a patient with reversal of portal flow due to portal hypertension or TIPS), any part of the liver, including benign parenchyma, has intense arterial "uptake". Moreover, no cancer and no part of the liver, literally takes up contrast agents in the arterial phase. Even contrast agents that eventually are transported into liver cells via membrane transporters do not enter cells in any meaningful concentration during the arteial phase. Thus the wording, "intense arterial uptake", is pathophysiolgicaly incorrect and cannot be considered appropriate.

The EASL 2012 guidelines [3] as well as the Spanish guidelines [4] utilized similar imperfect terminology as the AASLD guidelines 
("hypervascular in the arterial phase with washout in the portal venous or delayed phases" and "radiological hallmark, i. e. contrast uptake in the arterial phase and washout in the venous/late phase" or respectively "una captación de contraste en fase arterial seguido de lavado en fases venosas"). Also the Korean guidelines [5] ("hypervascularity in the arterial phase") and Japanese guidelines [6] ("intense arterial enhancement") adopt similar suboptimal definitions. The Italian position paper in 2013 [7] defined more clearly the diagnostic pattern in connection to both the arterial and portal patterns (homogeneous hyper-enhancement of the lesion in the arterial phase, followed by hypo-enhancement in the venous or delayed phase), but created confusion in its accompanying figure where it reported, "typical feature: wash-in and wash-out". More properly, the term "wash-in" should be used to refer to the phase of arrival of the contrast during the arterial phase, up to reaching the peak signal intensity [8], but not to describe the degree of contrast enhancement. Using the term "wash-in" to describe the degree of arterial-phase enhancement is inappropriate and should not be considered synonymous of hyperenhancement.

The term wash-out may cause similar confusion. For example, the term "wash-out phase" refers to the time period between peak contrast enhancement intensity and disapperance of contrast [8] - i. e., the phase during which de-enhancement occurs, not the degree of de-enhancement. Moreover, some investigators have used "washout" to refer to a lesion that hyperenhances in the arterial phase relative to the surrounding parenchyma and that becomes isoenhancing in the venous phase. The use of "washout" in this context is justifiable in the sense that the lesion did de-enhance relative to the surrounding parenchyma. However, this temporal enhancement pattern - arterial phase hyperenhancement followed by venous phase isoenhancement - is not specific for HCC. For this reason, the term "wash-out pattern" should be reserved for lesions that, after showing iso or hyperenhancement in the arterial phase, become hypoenhancing in the venous/delayed phase in comparison to the surrounding parenchyma. Accordingly the Canadian guidelines [9] define the "classical radiographic appearance of HCC as: a lesion that exhibits higher signal intensity than the surrounding liver in the arterial phase of a contrast-enhanced study and lower signal intensity than the surrounding liver in the venous or the delayed phase of the contrast examination (so-called portal venous 'washout')".

To summarize very few guidelines have provided optimal terminology for the hallmark imaging criteria of HCC.

In recent years, the American College of Radiology has supported the development of the Liver Imaging Reporting And Data System (LI-RADS ${ }^{\circledR}$ ) with the aim, among others, to establish consistent, rigorous, and precisely defined terminology for clinical care and research. The LI-RADS ${ }^{\circledR}$ definition of the hallmark enhancement pattern at CT, MRI and CEUS is "arterial phase hyperenhancement (in whole or in part, not rim or peripheral discontinuous globular enhancement) in conjunction with washout appearance" (https://www.acr.org/quality-safety/resources/ lirads), which is in keeping with the clear definitions expressed by the Canadian [9] and Italian [7] documents. Importantly, LI-RADS specifies that at CEUS (see scheme at https://www.acr.org/quality-safety/resources/lirads), the washout has to be of mild degree and late, namely starting after 60 seconds, in keeping with recent
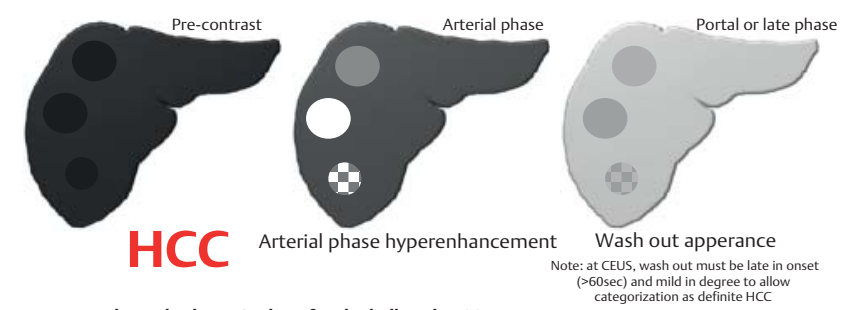

Proposed standard terminology for the hallmark HCC pattern: Arterial phase hyperenhancement (APHE) followed by wash-out appearance
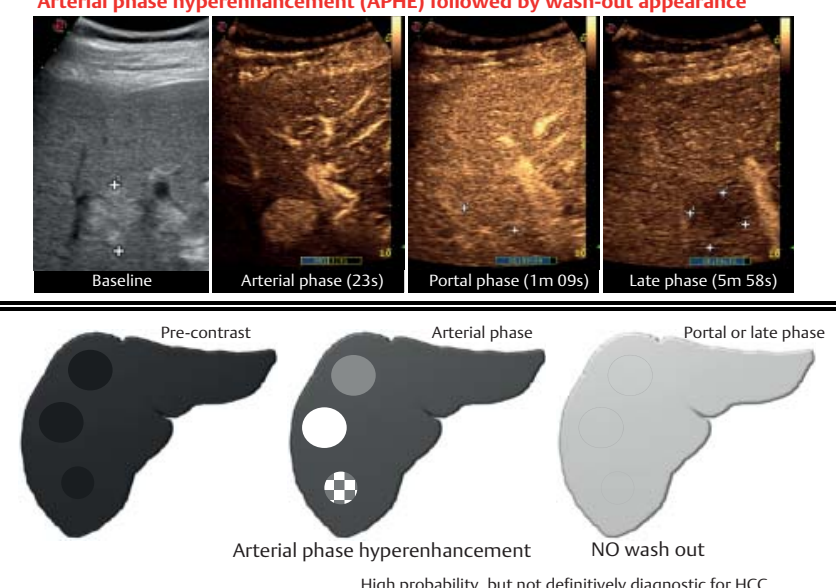

High probability, but not definitively diagnostic for HCC

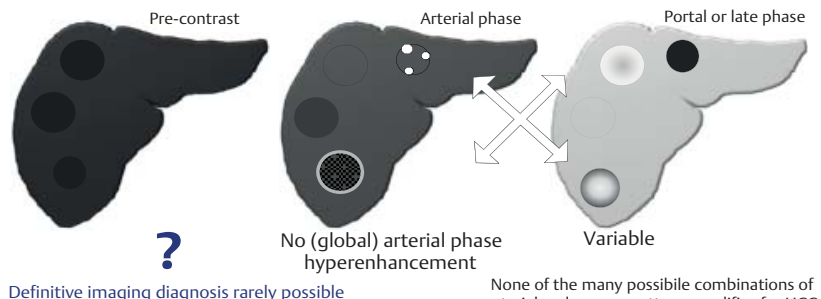
None of the many possibile combinations of

- Fig. 1 Schematic depictions of the patterns of appearance of focal lesions in cirrhosis in the arterial and venous contrast enhancement phases. The diagnostic pattern of HCC is shown, including an example at contrast enhanced ultrasound, in the upper half of the figure: this pattern is herewith proposed to be unequivocally and definitively defined as "arterial phase hyperenhancement (APHE) followed by wash-out appearance", according to the LI RADS definition of LR5.

publications [10 - 12] to prevent the misdiagnosis of cholangiocellular carcinoma as HCC [13-17].

Hence, we hereby propose the following as standardized terminology to describe the hallmark temporal enhancement pattern of HCC: "arterial phase hyperenhancement (APHE) followed by washout appearance". We also propose that the terms arterial phase hyperenhancement and washout appearance be defined as: arterial phase hyperenhancement = enhancement (of the lesion) in the arterial phase that is unequivocally greater than that of the liver; washout appearance = visually assessed temporal reduction in enhancement of the lesion relative to the liver from an earlier to a later phase resulting in portal venous phase hypoenhancement or delayed phase hypoenhancement.

This pattern is typical and diagnostic of HCC (with the additional requirement of mild and late occurrence of hypoenhancement = wash-out for CEUS) ( $\vee$ Fig. 1). According to LI-RADS, other imaging feature combinations such as APHE plus capsule appear- 
ance also can establish the diagnosis of HCC at CT or MRI, especially since capsule appearance can create the visual perception of "washout" in HCCs without true "washout", but this, as well as the pattern in the postvascular phase of Sonazoid ${ }^{\circledR}$, are beyond the scope of the present editorial, which focuses on vascular phases enhancement characteristics.

\section{Conflict of interest}

The authors declare the following conflict of interest: Fabio Piscaglia: Speaker fees: Bayer, Bracco; Advisory board: Bayer; Research contract: Esaote. Member of the board of directors of the International Contrast Ultrasound Society ICUS. Claude Sirlin: Industry grant support: Siemens, GE, Gerber; Consulting and service agreements: Bracco. Kwang-Hyub Han: nothing to disclose. Masatoshi Kudo: Speaker fees: Bayer, Eisai, MSD, Ajinomoto, Grants: Chugai, Otsuka, Takeda, Taiho, Sumitomo Dainippon, Daiichi Sankyo, MSD, Eisai, Bayer, Abbvie Advisory Consulting: Kowa, MSD, BMS, Bayer, Chugai, Taiho.

\section{References}

[1] Bruix ], Sherman M. Management of hepatocellular carcinoma. Hepatology 2005; 42: $1208-1236$

[2] Bruix J, Sherman M. Management of hepatocellular carcinoma: an update. Hepatology 2011; 53: 1020-1022

[3] European Association For The Study Of The L, European Organisation For $R$, Treatment Of C. EASL-EORTC clinical practice guidelines: management of hepatocellular carcinoma. J Hepatol 2012; 56: 908-943

[4] Forner A, Reig M, Varela M et al. Diagnóstico y tratamiento del carcinoma hepatocelular. Actualizacióndel documento de consenso de la AEEH, SEOM, SERAM, SERVEl ySETH. Med Clin (Barc) 2016; 146: 511.e1-511.e22

[5] Korean Liver Cancer Study G, National Cancer Center K. 2014 Korean Liver Cancer Study Group-National Cancer Center Korea practice guideline for the management of hepatocellular carcinoma. Korean J Radiol 2015; 16: 465-522

[6] Kokudo N, Hasegawa K, Akahane M et al. Evidence-based Clinical Practice Guidelines for Hepatocellular Carcinoma: The Japan Society of
Hepatology 2013 update (3rd JSH-HCC Guidelines). Hepatol Res 2015; 45: DOI: 10.1111/hept.12464

[7] Panel AE, Italian Association for the Study of the L, Committee AC et al. Position paper of the Italian Association for the Study of the Liver (AISF): the multidisciplinary clinical approach to hepatocellular carcinoma. Dig Liver Dis 2013; 45: 712-723

[8] Dietrich CF, Averkiou MA, Correas JM et al. An EFSUMB introduction into Dynamic Contrast-Enhanced Ultrasound (DCE-US) for quantification of tumour perfusion. Ultraschall in Med 2012; 33: 344- 351

[9] Burak KW, Sherman M. Hepatocellular carcinoma: Consensus, controversies and future directions. A report from the Canadian Association for the Study of the Liver Hepatocellular Carcinoma Meeting. Can J Gastroenterol Hepatol 2015; 29: 178-184

[10] Yuan MX, Li R, Zhang XH et al. Factors Affecting the Enhancement Patterns of Intrahepatic Cholangiocarcinoma (ICC) on Contrast-Enhanced Ultrasound (CEUS) and their Pathological Correlations in Patients with a Single Lesion. Ultraschall in Med 2016; 37: 609-617

[11] Schellhaas B, Wildner D, Pfeifer L et al. LI-RADS-CEUS - Proposal for a Contrast-Enhanced Ultrasound Algorithm for the Diagnosis of Hepatocellular Carcinoma in High-Risk Populations. Ultraschall in Med 2016; 37: $627-634$

[12] Liu G], Wang W, Lu MD et al. Contrast-Enhanced Ultrasound for the Characterization of Hepatocellular Carcinoma and Intrahepatic Cholangiocarcinoma. Liver Cancer 2015; 4: 241 - 252

[13] Galassi M, lavarone M, Rossi S et al. Patterns of appearance and risk of misdiagnosis of intrahepatic cholangiocarcinoma in cirrhosis at contrast enhanced ultrasound. Liver Int 2013; 33: $771-779$

[14] Vilana R, Forner A, Bianchi L et al. Intrahepatic peripheral cholangiocarcinoma in cirrhosis patients may display a vascular pattern similar to hepatocellular carcinoma on contrast-enhanced ultrasound. Hepatology 2010; 51: 2020-2029

[15] Salvatore V, Gianstefani A, Negrini G et al. Imaging diagnosis of hepatocellular carcinoma: recent advances of contrast-enhanced ultrasonography with SonoVue ${ }^{\circledR}$. Liver Cancer 2016; 5: 55-66

[16] Wildner D, Bernatik T, Greis C et al. CEUS in hepatocellular carcinoma and intrahepatic cholangiocellular Carcinoma in 320 patients - early or late washout matters: a subanalysis of the DEGUM multicenter trial. Ultraschall in Med 2015; 36: $132-139$

[17] Wildner D, Pfeifer L, Goertz RS et al. Dynamic contrast-enhanced ultrasound (DCE-US) for the characterization of hepatocellular carcinoma. Ultraschall in Med 2014; 35: 522-527 